Check for updates

Cite this: RSC Adv., 2019, 9, 9180

Received 5th December 2018

Accepted 21st February 2019

DOI: $10.1039 / c 8 r a 10013 f$

rsc.li/rsc-advances

\section{Effects of a low-strength magnetic field on the characteristics of activated sludge for membrane fouling mitigation}

\begin{abstract}
Ren Zhijun, (D) *abc Wang Pengfei, ${ }^{a}$ Tian Jiayu ${ }^{a}$ and Zhang Zhiliu ${ }^{b}$
This study aims to investigate the performance of a low-strength magnetic field in membrane bioreactors (MBRs) for membrane fouling mitigation and its effects on sludge characteristics and microbial community. The continuous operation of MBR with magnetic powder (MP-MBR) is denoted as the control-MBR, and a magnetic field is added to the MP-MBR to form the magnetic-MBR (M-MBR). The comparison between MP-MBR and M-MBR was conducted to treat synthetic wastewater. The results showed that the application of a low-strength magnetic field not only decreased the zeta potential and increased the particle size, but also improved the dehydrogenase activity and stimulated microbes to produce fewer SMPs, which markedly contributed to the improved filtration performance of MBRs. The variations in the microbial communities from the two MBRs at genus levels confirmed that the addition of a low-strength magnetic field significantly affected the microbial community and composition, further altered the microbial metabolites, and consequently affected the membrane fouling evolution.
\end{abstract}

\section{Introduction}

Membrane fouling, which substantially reduces the flux permeability and thus deteriorates the filtration performance of membranes, is an inevitable phenomenon that occurs during the application of membrane bioreactors (MBRs). ${ }^{\mathbf{1} 2}$ Recently, magnetic technology combined with membrane bioreactors (MBRs) has garnered attention from researchers. Liu et al. ${ }^{3}$ and Ren et al. ${ }^{4}$ reported that the size of the aerobic granular sludge with magnetic seeds increased as the particle size increased and the membrane fouling rate was significantly reduced by adding magnetic species. Magnetic bio-effect is considered to be the main factor in mitigating membrane fouling when magnetic powder is added in membrane bioreactors (MBRs) for membrane fouling mitigation. ${ }^{5}$ Wang et $a .^{6}{ }^{6}$ reported that magnetic powder improved the dehydrogenase activity, enhanced the biomass growth, and exhibited good performance in the mitigation of membrane fouling. Chen et al. ${ }^{7}$ found that there are a variety of microbial species in the reactor and that the membrane fouling rate can be decreased by adding magnetic particles to the MBR.

Compared with other fouling mitigation techniques, the advantages of a magnetic-MBR include greater flexibility, less chemical usage, excellent effluent quality, ${ }^{8}$ and the ability to

${ }^{a}$ School of Energy and Environmental Engineering, Hebei University of Technology, Tianjin, 300130, China. E-mail: renzhijun2003@126.com

${ }^{b}$ College of Aerospace and Civil Engineering, Harbin Engineering University, Harbin, 150001, China

${ }^{c}$ State Key Laboratory of Urban Water Resource and Environment, Harbin Institute of Technology, Harbin 150090, China counter fouling by simultaneously integrating membrane filtration, magnetism, and biological treatment into a single system. ${ }^{9,10}$ In recent years, the physical, chemical and biological effects of magnetic fields on the microbial degradation process have been widely studied in biologically activated sludge. ${ }^{\mathbf{1 1 , 1 2}}$ Both the growth of microorganisms $\mathbf{1 3}^{\mathbf{1 3}, \mathbf{1 4}}$ and the biodegradation ability of microorganisms could be affected by magnetic field. ${ }^{15}$ However, the knowledge of the sludge properties, microbial community as well as their connection to membrane fouling by a magnetic field, especially low-intensity magnetic field, in MBRs is still limited. Also, the anti-fouling mechanisms of lowintensity magnetic field, fulfilled by magnetic bioeffects, have not been well examined and still need to be verified.

In this study, a permanent magnet was applied to a membrane bio-reactor (MBR) with magnetic activated sludge (MP-MBR) to form a magnetic-MBR (M-MBR). A comparison was conducted to evaluate the performance of M-MBR and MP-MBR based on sludge properties and membrane fouling. In order to link the microbial community and membrane fouling, highthroughput sequencing technology served to compare the microbial communities of bulk sludge and biofilm sludge in both MBRs at the genus level and possible anti-fouling mechanisms of a low-strength magnetic field were also examined and discussed.

\section{Materials and methods}

\subsection{Operation of MBRs}

In order to better understand the mechanism of a magnetic field on membrane fouling control, parallel experiments of $\mathbf{M}$ - 
MBR and MP-MBR were investigated. The experimental apparatus is shown in Fig. 1.

The CODcr of the wastewater was approximately $500 \mathrm{mg} \mathrm{L}^{-1}$. The operation of both the MBRs was as follows: the wastewater was fed into the reactor from the original water tank using a peristaltic pump, and an electronic timer was used to maintain a 10 minute water cycle that included a run time of 8 minutes and a stop time of 2 minutes, in which the content in the effluent was constant, the membrane flux was $25 \mathrm{~L}\left(\mathrm{~m}^{-2}\right.$ $\mathrm{h}^{-1}$ ), the hydraulic retention time (HRT) was $4.8 \mathrm{~h}$, the aeration was continuous, the dissolved oxygen was approximately $3 \mathrm{mg} \mathrm{L}^{-1}$, the temperature inside the reactor was kept between $15{ }^{\circ} \mathrm{C}$ and $25{ }^{\circ} \mathrm{C}$, and the $\mathrm{pH}$ value was maintained between 6.5 and 7.5. A vacuum gauge was installed in the outlet pipe to monitor the TMP, and the membrane was cleaned when the TMP reached a critical value of 0.05 .

In the M-MBR process, two permanent magnets on a turntable were symmetrically placed on both sides of the center of the flat film, which has dual purposes: directly influencing microbial activities and controlling the magnetic powder movement in MBR process. The length, width and height of the permanent magnets were $100 \mathrm{~mm}, 100 \mathrm{~mm}$ and $10 \mathrm{~mm}$, respectively. The intensity of the magnetic field could be adjusted from $0 \mathrm{mT}$ to 45 $\mathrm{mT}$ by regulating the distance from the turntable. The rotation rate of the turntable was controlled by the electronic timer, and the rotary run time: down time were $1 \mathrm{~min}: 2 \mathrm{~min}, 2$ $\min : 2 \mathrm{~min}$, and $3 \mathrm{~min}: 2 \mathrm{~min}$. Based on the organic removal efficiency and the change in the MLSS and TMP under different magnetic field intensities and rotary run time : down time values, a magnetic field strength of $35 \mathrm{mT}$ and a rotary run time : down time of 2 min : 2 min were determined as the optimal experimental conditions. The results are not shown in this paper. ${ }^{16}$

Activated sludge was collected from the secondary clarifier of the Daqing Oil Sewage Treatment Plant in Heilongjiang, China. The MLSS concentration was kept constant at approximately $16000 \mathrm{mg} \mathrm{L}^{-1}$.

A frame plate membrane module from Shanghai Snapp Membrane Separation Technology Co., Ltd., was used. The

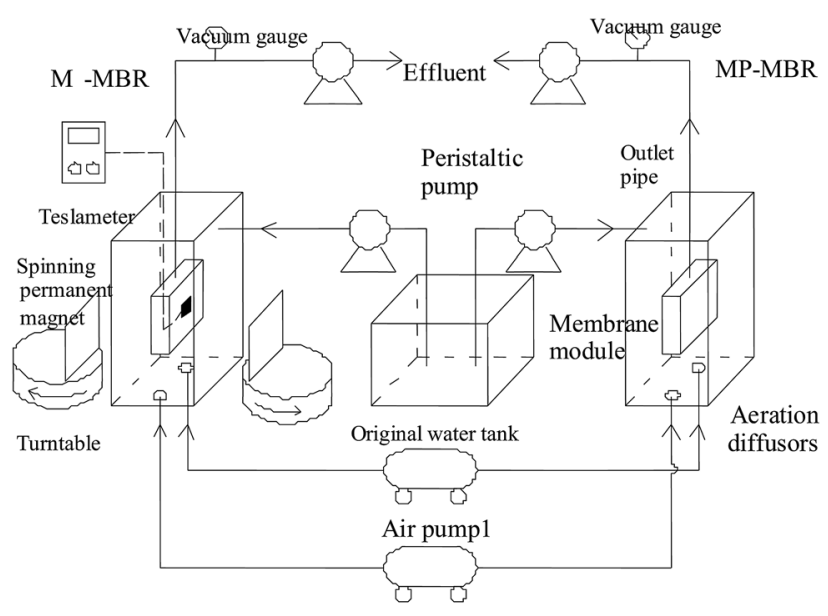

Air pump2

Fig. 1 A schematic diagram of MBR. membrane was composed of polyvinylidene fluoride (PVDF) and had a total area of $0.1 \mathrm{~m}^{2}$ and a pore size of $0.1 \mu \mathrm{m}$.

$\mathrm{Fe}_{3} \mathrm{O}_{4}$ powder, which is a common magnetic material with weak magnetic properties and strong adsorption ability due to its surface area, was used in this study. The powder size ranged from $1 \mu \mathrm{m}$ to $12 \mu \mathrm{m}$, with $50 \%$ within the size range of $2-4 \mu \mathrm{m}$. The magnetic powder was pretreated as follows: $20 \mathrm{~g}$ of magnetic powder (with the $\mathrm{Fe}_{3} \mathrm{O}_{4}$ content greater than 98\%) was added to a $1 \mathrm{~L}$ beaker and mixed with deionized water. The magnetic powder was allowed to settle for 30 minutes under the action of a round magnet placed in the bottom of the beaker, and then the supernatant was removed. The settling step was repeated twice, and then the beaker was placed in an oven at $105{ }^{\circ} \mathrm{C}$ for $24 \mathrm{~h}$. Before it was added to the MBR process, the magnetic powder was transferred to a $1 \mathrm{~L}$ beaker, which was placed on the permanent magnet for 30 minutes to magnetize the magnetic powder. ${ }^{17}$

A quantification approach, indicated by mean SD, was used. Data in the figures are mean \pm SD values of 3 replicates and mean was used for data analysis and comparison.

\subsection{Analysis methods}

All effluent samples were filtered through $0.45 \mu \mathrm{m}$ membrane filters (mixed cellulose ester, Advantec) before chemical analysis. The MLSS concentration was measured using the standard method. ${ }^{18}$ Dehydrogenase was measured by following the Tracey method. ${ }^{19}$ The zeta potential of the supernatant was measured using a Zeta meter (ZetaSizer3000, England). The membrane samples were analyzed using attenuated total reflectance Fourier transform infrared (ATR-FTIR) spectroscopy (Spectrum One, Perkin Elmer) to characterize the chemical functional groups of the dried sludge samples. The pretreatment process for sample preparation began with the slow removal of the loose sludge from the membrane surface using water, cleaning the surface gel layer of the diaphragm using deionized water, and drying the mixed liquid in a drying oven at $105{ }^{\circ} \mathrm{C}$. The surface contaminants from the above procedure was mixed with $\mathrm{KBr}$ powder $(1: 100)$, which was dried to a constant weight in a vacuum dryer and pressed into pellets using a tablet press. The spectra were recorded over the wavelength range of $4000 \mathrm{~cm}^{-1}$ to $400 \mathrm{~cm}^{-1}$.

Proteins and polysaccharides are the largest components of SMPs; therefore, they can be used to infer the SMP content. The amounts of polysaccharides were measured using the anthrone-sulfuric acid method and those of the proteins were measured via UV-vis spectrophotometry.

The activated sludge samples were collected from the seed sludge and the biofilm samples were taken from the fouled membranes using a sterile steel knife. After DNA extraction and PCR amplification, the microbial communities were analyzed by Illumina MiSeq sequencing.

\section{Results and discussion}

After 3 months of operation, both the MBR processes exhibited a stable COD removal performance. Magnetic powder 


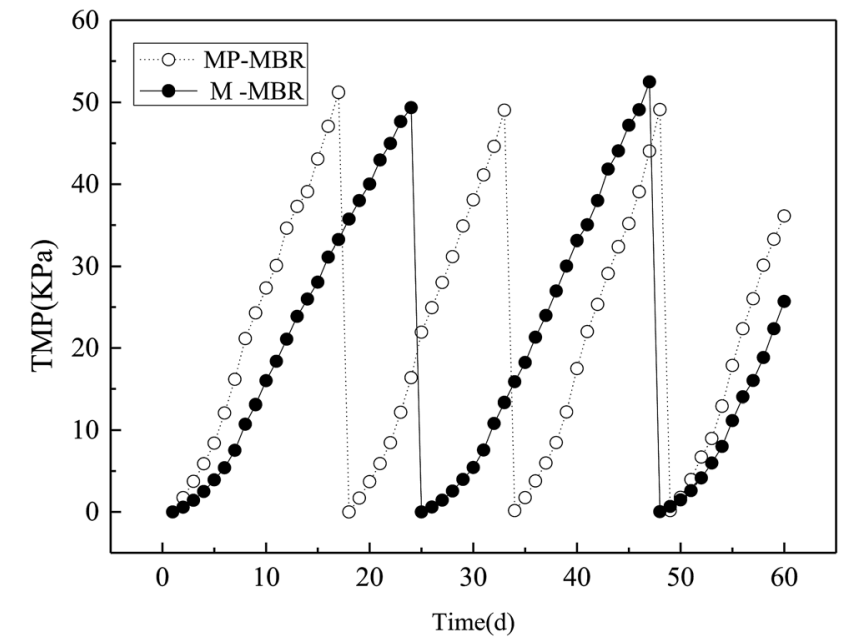

Fig. 2 TMP profile for each MBR.

(650 $\mathrm{mg} \mathrm{L}^{-1}$ ) was added to one of the MBR reactors to form an MP-MBR; magnetic powder with the same dosage and two permanent magnets was applied to another MBR to form an M-

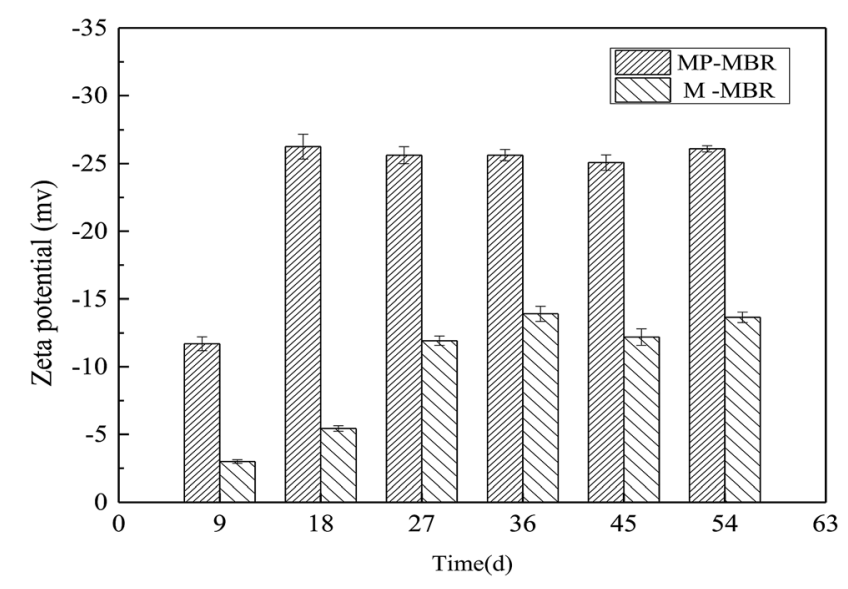

Fig. 3 Zeta potential of activated sludge mixed liquor.
MBR. Both reactors were operated under the same conditions (details are given in Section 2.1).

\subsection{The performance of two MBRs}

Fig. 2 shows the discrepancies in the TMP values of the M-MBR and the MP-MBR (control-MBR) during 60 days of operation. The TMP in the M-MBR reached above $50 \mathrm{kPa}$ until day 17 with an average fouling rate of TMP of $3.01 \mathrm{kPa} \mathrm{d}^{-1}$, while the TMP in the M-MBR reached above $50 \mathrm{kPa}$ until day 24 with an average fouling rate of TMP of $2.06 \mathrm{kPa} \mathrm{d}^{-1}$. A similar result was reported by Liu et al. ${ }^{5}$ The significantly low fouling rate in the MMBR indicates that integrating the magnetic field unit into an MBR system is a promising method to alleviate the negative effects of membrane fouling, thereby prolonging the operation periods for MBRs.

\subsection{Zeta potential and particle sizes of mixed liquor colloid}

Zeta potential is an important indicator of membrane fouling. During the operation period, the value of the zeta potential of the two reactors decreased in the early stage and remained at a lower level in the later stage, with a small fluctuation. The zeta potential of the MP-MBR changed from $-\mathbf{1 1 . 7} \mathrm{mV}$ to $-26.255 \mathrm{mV}$, with a mean value of $-23.395 \mathrm{mV}$, while the zeta potential of the M-MBR changed from $-2.989 \mathrm{mV}$ to $-13.917 \mathrm{mV}$, with a mean value of $-10.020 \mathrm{mV}$. The value of the zeta potential of the M-MBR was $57.17 \%$ smaller than that of the MP-MBR, which indicated that the magnetic field played a very significant role in decreasing the zeta potential in the mixed liquor colloid. Zhou et al. ${ }^{20}$ in their study, documented similar results and found that the maximum sludge zeta potential reduction ( $46.5 \%$ to $51.4 \%$ ) was achieved when the center of the magnetic field was approximately $0.4 \mathrm{~T}$. Li et al. ${ }^{21}$ also found that the zeta potential of the sludge was greatly influenced by a parallel magnetic field and that the largest reduction was to 46.5\% (Fig. 3).

One cause of the difference in membrane fouling rates was the variation in the distribution of particle sizes in the mixed
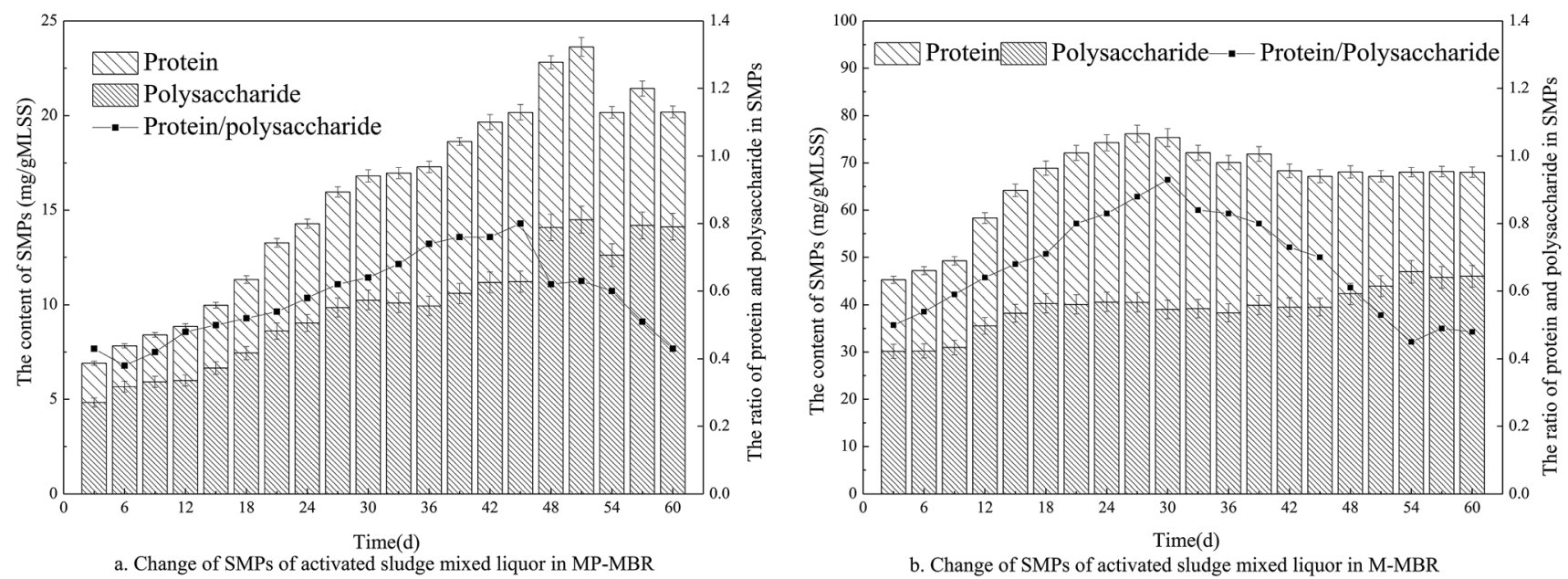

Fig. 4 Change in the content of SMPs of activated sludge mixed liquor. 


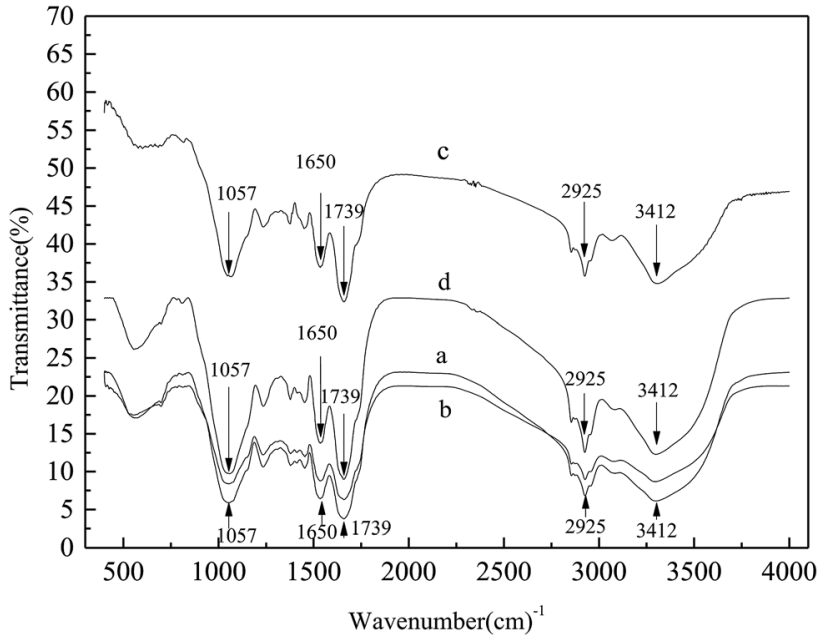

Fig. 5 FTIR spectra of sludge and cake layer in the control-MBR and M-MBR. (a) Activated sludge mixed liquor in M-MBR. (b) Activated sludge mixed liquor in MP-MBR. (c) The cake layer on the membrane surface in M-MBR. (d) The cake layer on the membrane surface in MPMBR.

liquor. The average particle sizes of the sludge in the two reactors were detected. In the control-MBR, the particle sizes of the sludge changed from $117.32 \mu \mathrm{m}$ to $162.09 \mu \mathrm{m}$, which was not a significant increase. The size of the particles in the sludge in the M-MBR was always larger than that in the control-MBR, which increased evidently from $146.22 \mu \mathrm{m}$ to $280.17 \mu \mathrm{m}$. There was a marked increase in the particle size from $28.89 \mu \mathrm{m}$ to $118.08 \mu \mathrm{m}$, showing that the application of a magnetic field could significantly increase the sludge particle size.

\subsection{Effect of a low-strength magnetic field on SMP}

According to previous reports, SMPs have been found to play an important role in membrane fouling. ${ }^{22,23}$ Polysaccharides and proteins as the major components of SMP were analyzed periodically from the mixed liquors of each MBR.

According to Fig. 4, the SMP content in the two reactors increased with time, and as expected, the SMP content and the proportion of proteins and polysaccharides in the M-MBR remained lower than those in the MP-MBR. It is evident that polysaccharides are the dominant component in the SMPs in both reactors. The lower protein to polysaccharide ratio in the M-MBR implies better settleability due to the hydrophobic nature of proteins. SMPs have been found to play an important role in membrane fouling. ${ }^{24}$ It has been reported that the variations in EPS and SMP simply reflect changes in the microbial community. ${ }^{25}$ Consequently, it is necessary to compare microbial communities of both the MBRs.

To corroborate the degradation of SMPs in bound water in sludge due to adsorption and bio-oxidation, FTIR analysis was performed to determine the variations in the functional groups of the sludge samples and the cake layer. As shown in Fig. 5, peaks of the functional groups of proteins and polysaccharides were clearly distinguished in the FTIR spectra.

From the spectra in Fig. 5, the trends of the cake layer on the membrane surface and for a mixed liquid were always the same. There were five additional significant absorption peaks in the mixed liquid spectra, whose wavenumbers were $1057 \mathrm{~cm}^{-1}$, $1650 \mathrm{~cm}^{-1}, 1739 \mathrm{~cm}^{-1}, 2925 \mathrm{~cm}^{-1}$ and $3412 \mathrm{~cm}^{-1}$.

The $3412 \mathrm{~cm}^{-1}$ absorption peak was generated by the oscillatory contraction of the $\mathrm{O}-\mathrm{H}$ bond, possibly indicating the presence of organic acids. The $2925 \mathrm{~cm}^{-1}$ absorption peak was generated by the oscillatory contraction of the aliphatic $\mathrm{C}-\mathrm{H}$ bond, and the $1650 \mathrm{~cm}^{-1}$ absorption peak was generated by the oscillatory contraction of the $\mathrm{C}=\mathrm{N}$ bond and its amideassociated state, illustrating the existence of amide compounds; the structure of the amide compounds was the typical structure of proteins. ${ }^{26}$ The $1739 \mathrm{~cm}^{-1}$ absorption peak was generated by the carbonyl $\mathrm{C}=\mathrm{O}$ bond, which may be related to polysaccharides, and the $1057 \mathrm{~cm}^{-1}$ absorption peak was generated by methyl $\mathrm{C}-\mathrm{H}$ symmetric bending vibrations, which is very useful for the identification of methyl groups and may also be related to carbohydrates. ${ }^{27}$

The amount of substances in the cake layer and the mixture of the absorption peaks in the M-MBR were significantly less than those in the MP-MBR, showing that the amounts of organic acids, polysaccharides and proteins in the M-MBR were significantly less than those in the MP-MBR. This conclusion is consistent with the conclusions presented in Section 3.1. As a type of protein, dehydrogenase (DHA) in living organisms can realize the hydrogen atom activation of organic matter in the oxidation process, the transfer of energy and the promotion of material circulation. The activity of dehydrogenase in a mixed liquid in the M-MBR was always higher than that in the MPMBR. The dehydrogenase activity in the MP-MBR was between $41.27 \mathrm{mg} \mathrm{TF} \mathrm{g}^{-1}$ MLSS $^{-1}$ and $47.22 \mathrm{mg} \mathrm{TF} \mathrm{g}^{-1} \mathrm{MLSS} \mathrm{h}^{-1}$ and showed negligible changes, while the dehydrogenase activity in

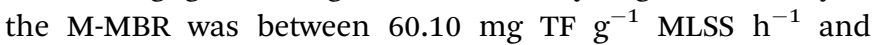
$63.32 \mathrm{mg} \mathrm{TF} \mathrm{g}^{-1} \mathrm{MLSS} \mathrm{h}^{-1}$, which was increased by $27.07 \%$ in the case without a magnetic field. Jung et al. ${ }^{28}$ showed that magnetic fields could enhance the rate of phenol

Table 1 Richness and diversity estimators of the bacterial phylotypes of bulk sludge and biofilm sludge in both MBRs

\begin{tabular}{|c|c|c|c|c|c|c|c|}
\hline Samples & Optimized sequences & OTUs & Shannon index & Ace index & Chao index & $\begin{array}{l}\text { Good's } \\
\text { coverage }\end{array}$ & Simpson \\
\hline HHY0 & 16139 & 1323 & 3.333 & 8408.230 & 4259.374 & 0.965 & 0.137 \\
\hline HHY1 & 18892 & 1774 & 4.015 & 9264.255 & 5246.579 & 0.954 & 0.078 \\
\hline HHY2 & 25573 & 1823 & 3.869 & 12669.457 & 6867.809 & 0.955 & 0.070 \\
\hline MO1 & 18950 & 1937 & 4.839 & 9752.440 & 5240.780 & 0.932 & 0.068 \\
\hline $\mathrm{MO} 2$ & 25249 & 2572 & 4.381 & 12802.746 & 7129.809 & 0.932 & 0.038 \\
\hline
\end{tabular}


biodegradation in the activated sludge mixed culture by $30 \%$. Ren et $a .^{29}$ found that lower magnetic fields (15-25 mT) enhanced the permeability of the cell membrane and improved the activity of the oil-degrading bacteria. Thus, the appropriate magnetic field intensity plays a role in promoting the metabolism of microorganisms. The magnetic field stimulated the synthesis of microbial cells and the secretion of more dehydrogenase; thus, the total amount of dehydrogenase in the system increased, and more substrate was consumed.

\subsection{Effect of a low-strength magnetic field on microbial community and composition}

3.4.1 Microbial diversity and richness. High-throughput sequencing served to characterize the microbial community and the composition in the MBRs, and provided deep insights
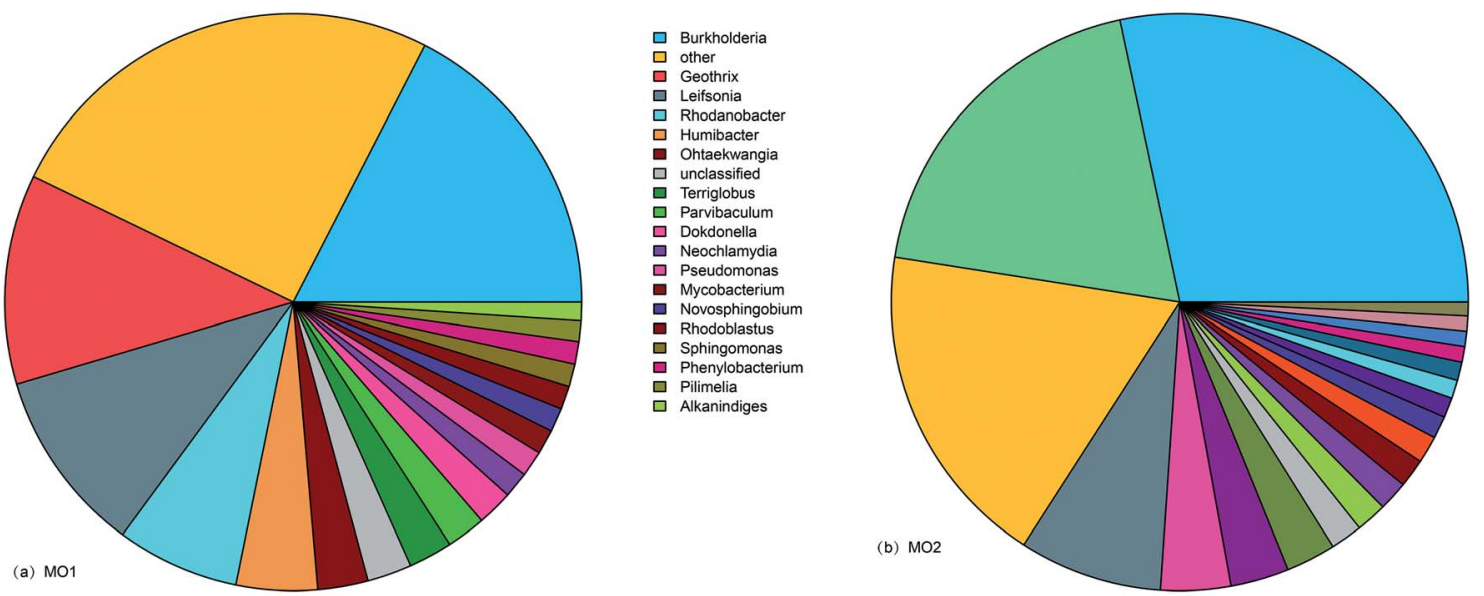

ㅁ Burkholderia ㅁ Pedobacter

Other

ㅁ Pseudomonas

Methylophilus

Mesorhizobium

Alkanindiges

Neochlamydia

Rhodoblastus

Novosphingobium

Enterobacter

- Rhizobium

- Phenylobacterium

Polaromonas

ㅁ Polaromonas

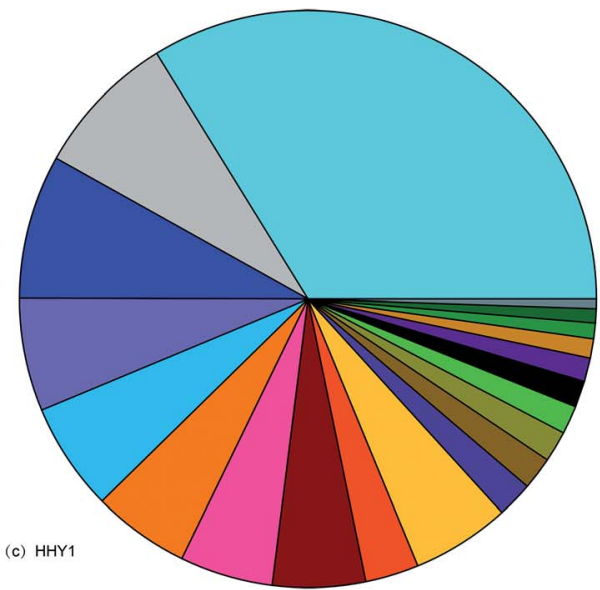

ㅁ Rhodanobacte Ferruginibact Rudaea

ㅁ Burkholderia

D Dokdonella

Rhodoblastus

ㅁ Edaph

Microbacterium

․ Rhodopila

ㅁ Pilimelia

ㅁ Parvibac

- Enterobacter

ㅁoultella

맘 1

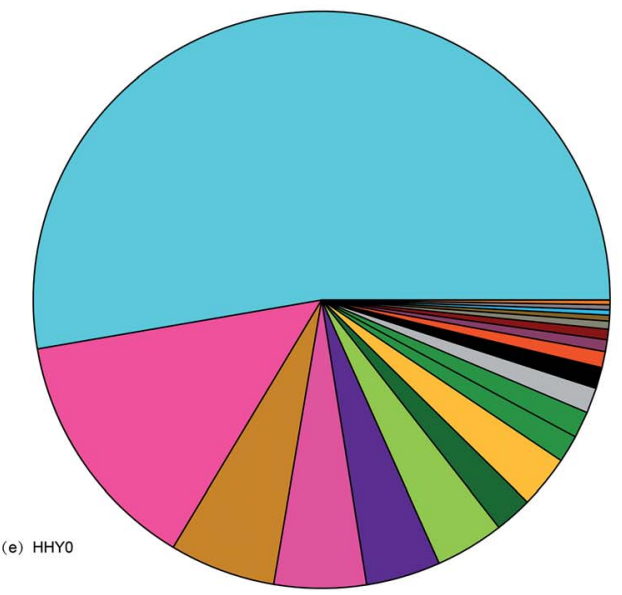

ㅁ Rhodanobacter

D Dokdonella

口 Raoultella

ㅁ Pseudomonas

ㅁ. Enterobacter

- Gp1

믈

ㅁ Terriglobus

Citrobacter

unclass

- Edaphobacter

口 Acidisoma

Rhodoblastus

Rulvimonas

Burkholderia

Roseomonas

Fig. 6 Taxonomic classification of the microbial community at the genus level. 
into the effect of a low-strength magnetic field on the microbial community and membrane fouling mitigation. The microbial population distribution in a mixed solution from the reactors and on the membrane surface was analyzed. (Note that in this section, MO1 represents the membrane surface in the MP-MBR, MO2 represents the membrane surface in the M-MBR, HHY1 represents the MP-MBR activated sludge mixed liquor, HHY2 represents the M-MBR activated sludge mixed liquor, and HHY0 represents the activated sludge mixed liquid without any material.) To reflect the abundance of each sample more intuitively, the parts with very low abundance were collectively presented as "other" in the chart; taking the genus level as an example, the abundance pie charts of the sample species were drawn.

After necessary denoising and filtering, the effective reads from each sample were used for further analyses. The diversity estimators of Chao Index, Ace Shannon, Simpson and Good's coverage for each sample are illustrated in Table 1. In this study, the Good's coverage of all samples was above 0.93 , indicating that the sequencing depth could reflect the real microbial community for both MBRs.

It was apparent that both the microbial richness (estimated by Chao 1 and Ace) and diversity (estimated by Shannon and Simpson) of bulk sludge and biofilm layer in M-MBR were higher than those in MP-MBR. Thus it could be inferred that the microbial community in M-MBR had greater richness and more diversity than that in MP-MBR, and further comparison of the microbial composition was conducted to reveal more information on the microbial community differences between those in the M-MBR and MP-MBR.

3.4.2 Comparison of microbial community at the genus level. To better understand the effect of introducing a lowstrength magnetic field on the microbial community and composition and the procedure to mitigate membrane fouling, the sequence reads obtained from Illumina MiSeq sequencing were systematically analyzed at the genus level.

Fig. 6 shows that some abundance differences existed among sample species at the genus level; the abundances of MO1, MO2, HHY1, HHY2, and HHY0 were 20, 23, 17, 18 and 15, respectively. From the viewpoint of the overall structure, MO1 and MO2, HHY1 and HHY2 were similar, but HHY0 was different from the others. The deep blue slices of the MO1 and MO2 pie charts represent the Burkholderia genus, which accounted for $17 \%$ of MO1 and $28 \%$ of MO2, $5 \%$ of HHY1 and $4 \%$ of HHY2, respectively; HHY0 did not contain this genus. The content of this genus on the membrane surface was much higher than that in the mixture. The members of the genus Burkholderia were formerly classified as Pseudomonas, whose surface has Gram-negative bacteria, high movability and aerobic rod-shaped bacteria. This genus plays a large role in the degradation of organic matter; thus, it is likely that this genus can degrade contaminants on the membrane surface. The red slice of the MO1 pie chart represents the genus of bacteria, whose proportion was $12 \%$, but there were no such bacteria in MO2 and the other samples; thus, there are currently only a few studies of this species, and the degradation of organic matter is not obvious. The green slice of the MO2 pie chart represents
Bacillus which accounted for $23 \%$ and only existed in MO2. And Bacillus is widely distributed in the reduction environment. These species are particularly helpful in organic matter degradation, which also explains the lesser membrane pollution in the M-MBR. The dark gray slices of the MO1 and MO2 pie charts represent the Lai Salmonella genus which accounted for $12 \%$ and $11 \%$, respectively. Although there were fewer studies on these bacteria, they played an irreplaceable role in the degradation of pollutants on the membrane surface in this study.

The above results show that a change in the microbial community occurs under a magnetic field..$^{30,31} \mathrm{Shi}^{32}$ found that a succession of alternate dominant species of different metabolic types was formed with an increase in the magnetic induction intensity. Shan $^{33}$ found that the magnetic effect increases the activity of some bacterial species, which adapt to the magnetic environment. The other species that are less adaptive are eliminated. The superior species will dominate as the variety decreases. These types of species are more likely to form the steady structure of the community. $\mathrm{Ni}^{34}$ also showed that denitrifying bacteria biomass under a constant magnetic field are higher in activated sludge samples.

\section{Conclusion}

This study provides a promising technique for significantly alleviating membrane fouling by introducing a low-strength magnetic field in MBRs from the two main findings: (1) the application of a magnetic field decreases the zeta potential and increases the particle size, which markedly contributes to the better filtration performance of MBRs; (2) the addition of a lowstrength magnetic field significantly affected the microbial community and composition, which has greater richness and more diversity in M-MBRs, and consequently affected the membrane fouling evolution.

\section{Conflicts of interest}

There are no conflicts to declare.

\section{Acknowledgements}

This study was supported by the National Natural Science Foundation of China (No. 51209053) and the Open Project of the State Key Laboratory of Urban Water Resource and Environment of the Harbin Institute of Technology (No. QA201619).

\section{References}

1 B. Lesjean, S. Rosenberger, C. Laabs, M. Jekel, R. Gnirss and G. Amy, Correlation between membrane fouling and soluble/ colloidal organic sub-stances in membrane bioreactors for municipal wastewater treatment, Water Sci. Technol., 2005, 51(6-7), 11-18.

2 L. Deng, W. Guo, H. H. Ngo, H. Zhang, J. Wang, J. Li, S. Xia and $\mathrm{Y} . \mathrm{Wu}$, Biofouling and control approaches in membrane bioreactors, Bioresour. Technol., 2016, 221, 656-665. 
3 A. P. Liu, Z. Y. Chen, K. M. Li and S. Guo, Cultivation of aerobic granular sludge with magnetic seed and its effect on membrane fouling in MBR, China Water Wastewater, 2010, 26, 1-4.

4 Z. J. Ren and J. C. Shao, Mechanism of Membrane Fouling Control in the Magnetic Activated Sludge (MAS) Process, Desalin. Water Treat., 2017, 66, 111-116.

5 Y. Liu, Q. Liu, J. Li, H. H. Ngo, W. Guo, J. Hu, M. T. Gao, Q. Wang and Y. Hou, Effect of magnetic powder on membrane fouling mitigation and microbial community/ composition in membrane bioreactors (MBRs) for municipal wastewater treatment, Bioresour. Technol., 2017, 249(10), 377-385.

6 H. Wang, Z. Chen, J. Miao and Y. Li, A novel approach for mitigation of membrane fouling: concomitant use of flocculant and magnetic powder, Bioresour. Technol., 2016, 209, 318-326.

7 Q. Chen, W. Nong and W. Cui, Mechanism and Application of Magnetic Technology in Wastewater Treatment, J. Environ. Sci. Manage., 2012, 8, 110-114.

8 G. U. Semblante, S. D. R. Tampubolon, L. Zhang, S. J. You, Y. F. Lin, T. C. Chang and F. C. Yen, Fouling reduction in membrane reactor through magnetic particles, J. Membr. Sci., 2013, 435(10), 62-70.

9 E. Illes and E. Tombacz, The role of variable surface charge and surface complexation in the adsorption of humic acid on magnetite, Colloids Surf., A, 2003, 230(1-3), 99-109.

10 L. Ai, C. Zhang and Z. Chen, Removal of methylene blue from aqueous solution by a solvothermal-synthesized graphene/magnetite composite, J. Hazard. Mater., 2011, 192(3), 1515-1524.

11 Q. X. Han and F. Q. Shao, The Increasing effect of magnetic field on wastewater treatment by activated sludge process, $J$. Fushun Pet. Inst., 2002, 8-10.

12 F. Ma, Q. Wang and X. S. Zhu, Application Status and Development Trend of Magnetic Technology in Wastewater Treatment, China Water Wastewater, 2010, 26(14), 34-37.

13 Z. Zhou, B. X. Bian and S. Li, Magnetic treatment for lowering zeta potential of biological sludge, Environ. Pollut., 2007, 29, 174-177.

14 M. Lebkowska, A. Rutkowska-Narozniak, E. Pajor, A. Tabernacka and M. Zaleska-Radziwill, Impact of a static magnetic field on biodegradation of wastewater compounds and bacteria recombination, Environ. Sci. Pollut. Res., 2018, 25(23), 22571-22583.

15 H. Yavuz and S. S. Celebi, Effects of magnetic field on activity of activated sludge in wastewater treatment, Enzyme Microb. Technol., 2000, 26(1), 22-27.

16 Z. L. Zhang, Analysis on Efficiency of Magnetic Field Improved MP-MBR for Vessel Oily Wastewater Treatment and Membrane Fouling Control, Harbin Engineering University, 2016.

17 W. F. Yuan, B. S. Liu and Z. N. Wang, Purifying Oil-Bearing Sewage Water By Using Magnetic Powder, Environ. Chem., 1991, 4, 12-18.

18 A. D. Eaton, L. S. Clesceri and A. E. Greenberg, Standard Methods for the Examination of Water and Wastewater,
American Public Health Association, Washington, DC, 2005, pp. 20001-23710.

19 T. Burdock, M. Brooks, A. Ghaly and D. Dave, Effect of assay conditions on the measurement of dehydrogenase activity of using triphenyl tetrazolium chloride, Adv. Biosci. Biotechnol., 2011, 2(4), 12.

20 S. Li, B. X. Bian and Z. Zhou, Study on Effect of Magnetic Treatment on Sludge Dewatering Ability, Res. Environ. Sci., 2007, 20(3), 119-123.

21 J. Li, Y. L. Yi, Y. Jiao, D. G. Zhang, Ch. L. Yuan, X. L. Cheng and J. D. Sun, Effect of magnetic field on soil microbes and soil enzyme activities in brown earth, Chin. J. Soil Sci., 2007, 38, 957-961.

22 S. Rosenberger and M. Kraume, Filterability of activated sludge in membrane bioreactors, Desalination, 2002, 146(1-3), 373-379.

23 J. Li, F. Yang, Y. Li, F. S. Wong and H. C. Chua, Impact of biological constituents and properties of activated sludge on membrane fouling in a novel submerged membrane bioreactor, Desalination, 2008, 225(1-3), 356-365.

24 S. Rosenberger, H. Evenblij, S. Poele, T. Wintgens and C. Laabs, The importance of liquid phase analyses to understand fouling in membrane assisted activated sludge processes-six case studies of different European research groups, J. Membr. Sci., 2005, 263(1-2), 113-126.

25 Y. Ding, Y. Tian, J. Liu, N. Li, J. Zhang, W. Zuo and Z. Li, Investigation of microbial structure and composition involved in membrane fouling in the forward osmosis membrane bioreactor treating anaerobic bioreactor effluent, Chem. Eng. J., 2016, 286, 198-207.

26 A. Omoike and J. Chorover, Adsorption to goethite of extracellular polymeric substances from Bacillus subtilis, Geochim. Cosmochim. Acta, 2006, 70(4), 827-838.

27 J. R. Pan, Y. C. Su, C. Huang and H. C. Lee, Effect of sludge characteristics on membrane fouling in membrane bioreactors, J. Membr. Sci., 2010, 349(1), 287-294.

28 J. Jung, B. Sanji, S. Godbole and S. Sofer, Biodegradation of Phenol: A comparative study with and without applying magnetic fields, J. Chem. Technol. Biotechnol., 2010, 56(1), 73-76.

29 Z. J. Ren, X. D. Leng, Z. X. Zhang and H. N. Feng, Effect of low-strength magnetic fields on the oil removal performance of oil-degrading microorganisms, Desalin. Water Treat., 2018, 120, 133-140.

$30 \mathrm{H}$. Chen, Research on PHAs production from activated sludge under the co-effect of "feast"/" "famine" condition and magnetic field, Zhejiang University, 2008.

31 R. Radhakrishnan and B. D. Kumari, Influence of pulsed magnetic field on soybean (Glycine max L.) seed germination, seedling growth and soil microbial population, Indian J. Biochem. Biophys., 2013, 50(4), 312-317.

32 B. Shi, Study on the effects of low-intensity steady magnetic field on wastewater treatment with biofilm reactor, Jinan University, 2010.

$33 \mathrm{~J}$. Shan, Effect of magnetic system in SBR-denitrification and the microbial community dynamic analysis, Harbin Institute of Technology, 2010.

$34 \mathrm{~J}$. Y. Ni, The effect of stationary magnetic field on the anaerobic activated sludge performance, Shandong University, 2012. 